\title{
Parental Report versus Standardized Test as Measures of Language Improvement After Intervention in Children at Risk for ADHD
}

\author{
Eliane Ramos \\ Department of Communication Sciences \& Disorders \\ Florida International University, Miami \\ Florida, USA \\ Mildred Suarez \\ Albizu University, Miami \\ Florida, USA \\ Katie C. Hart \\ Paulo A. Graziano \\ Center for Children and Families, Department of Psychology \\ Florida International University, Miami \\ Florida, USA
}

\begin{abstract}
This study examined the congruence between standardized testing and parental report in measuring language improvement in children at risk for ADHD after intervention. Twelve children ages 5 to 7 received speech-language therapy over an eight-week period. A standardized test and a parental report were administered pre and post intervention for comparison. Results indicated language improvement in both measures, with parental report more congruent with standardized testing pre intervention than post intervention. Implications for relying on parental reports for measuring intervention effectiveness are discussed.
\end{abstract}

Keywords: ADHD, language impairment, parental report, intervention effectiveness, preschool

\section{Introduction}

Approximately $6 \%$ to $8 \%$ of preschoolers show speech and language deficits unrelated to a known etiology such as behavioral or emotional difficulties and autism spectrum disorder (Law, Garrett, \& Nye, 2004). Evidence indicates that these children are at risk for challenges that go beyond school readiness and literacy to include emotional, behavioral, and social difficulties. Research also has shown that these difficulties may ultimately lead to long-term consequences into adulthood if children's needs are not met in early years (Bickford-Smith, Wijayatilake, Woods, 2005; Wake et al., 2013). Given such negative impacts on academic and social readiness, effective treatment of speech and language difficulties in the early years is of extreme importance.

In the last decade, there has been a rapid growth in studies examining treatment effectiveness of children with speech and language delays (Wake et al., 2013), but conclusions are still limited. Intervention results have suggested positive benefits in the areas of expressive vocabulary and syntax; however, these results are based mostly on the performance of individual children and very small groups (Broomfield \& Dodd, 2011). In a meta-analysis of 54 intervention trials, Wake et al. (2013) concluded that results were limited as small sample sizes, heterogeneity of participants, and few replications were evident, but that there was enough evidence of improvements in expressive language in the participating children (Broomfield \& Dodd, 2011; Wake et al. 2013).

Children with Attention-Deficit/Hyperactivity Disorder (ADHD) often show associated language impairments (Redmond, Ash, \& Hogan, 2015). ADHD is a neurodevelopmental disorder characterized by a triad of symptoms; inattention, hyperactivity, and impulsivity (Barkley, 2014). Impairments associated with ADHD affect 10 to $25 \%$ of young children (Voeller, 2004), are more prevalent in males than females, and are the most common reason for child mental health referrals (DuPaul, Weyandt, \& Janusis, 2011; Cormier, 2008). 
As part of the diagnostic process when evaluating young children with ADHD, particularly if communication delays or deficits are noted, the evaluator (e.g., pediatrician, psychologist, child psychiatrist) may refer the family to a speechlanguage pathologist (SLP) who will assess the child for language deficits as well as other developmental delays. Such comprehensive evaluation will aid in the decision-making process related to diagnosis, eligibility for services and the planning of treatment (Crais, 2011).

Children with ADHD are more likely to face significant impairments in academic functioning resulting in poorer grades, lower scores on standardized tests, and a greater need for special education in comparison to peers without the disorder (Redmond et al., 2015; DuPaul et al., 2011). A high comorbidity rate of approximately 45\% between ADHD and language impairment (LI) has been reported (Docking, Munro, Cordier, \& Ellis, 2013). For children with ADHD with an associated LI, the risks for academic difficulties are even higher and improved management of LI in these children would contribute to better academic outcomes(Redmond et al., 2015).

A study conducted by Sciberras et al. (2014) examined the impact of language difficulties on social and academic functioning of children with ADHD. Their study included 179 children with ADHD who were assessed using the Clinical Evaluation of Language Fundamentals-5 (CELF-5) (Wiig, Semel, \& Secord, 2013) screener as well as other screening/assessment tools to assess their academic/social functioning as well as nonverbal IQ. Children with concurrent ADHD and LI (as identified by the CELF-5) had poorer academic outcomes than those without an associated LI. As a result, the researchers suggested that children with ADHD who are falling significantly behind academically should be referred for language assessments (Sciberras et al., 2014).Yet, the researchers noted that standardized language tests are rarely included in established batteries of assessments for children with ADHD.

Although research reports have provided insight concerning assessment and treatment of ADHD, little evidence is available concerning the effectiveness of speech and language treatment with children at risk for ADHD within controlled intervention settings (Armstrong \& Nettleton, 2004; Oram, Fine, Okamoto\& Tannock, 1999). Parental reports are useful tools as they can provide insight into the language strengths and weaknesses of children that might not be easily assessed through standardized evaluations. For example, parental measures have shown that children with ADHD have more difficulty with conversational skills when compared to children without ADHD (Timler, 2014). In addition to giving insight into a child's pragmatic abilities, some studies have also examined the feasibility of using parental report measures to screen and identify a language impairment in children with ADHD. It is important to obtain information from parental measures regarding their children's language abilities not only to create the most effective speech and language goals, but also to compare parental information to that of standardized assessments both to diagnose the language impairment as well as to measure progress achieved in therapy.

Assessment drives important clinical decisions that help Speech-Language Pathologists (SLPs) arrive at appropriate conclusions and recommendations regarding the need, frequency, structure, and outcomes of intervention (Shipley \& McAfee, 2015). Roulstone, Wren, Bakopoulou, and Lindsay (2012) argued that implementation of specific types of intervention should be based on intervention outcomes, measured primarily on the performance of speech, language, and communication skills of individual children.Such measurement should usea variety of assessment tools to determine the presence and absence of a disorder and to compare treatment results. According to IDEA 2004 guidelines, no single tool should be used in assessments or evaluations. Instead, eligibility for services should be determined by the use of a variety of testing sources in multiple contexts as well as the clinician's clinical expertise (ASHA, 2008). The same assessment guidelines can be used to determine language outcomes due to intervention, including a variety of tools such asformal standardized tests or informal measures such as criterion-referenced tests, and parental reports.

For formal testing, an SLP may use a standardized norm-referenced test, most commonly used for evaluating articulation or language disorders, that allows for a comparison of an individual's performance to a normative group. A standardized test provides standard procedures in order to reduce test-giver bias and other extraneous influences that may affect the individual's performance (Shipley \& McAfee, 2015). It is important for SLPs to determine that standardized measures taken, especially for infants and toddlers, demonstrate psychometric soundness and strong evidence to support their quality (Crais, 2011). During assessment, children's language skills are often examined first. A common test used for the preschool age is the Preschool Language Scales, $5^{\text {th }}$ Edition (PLS-5) (Zimmerman, Steiner, Pond, 2011) which provides an auditory comprehension and expressive communication score. This test can be administered in a relatively short period of time, and is considered to have a high level of reliability and validity (Denman et al., 2017). 
On the other hand, criterion-referenced instruments (e.g., checklists or probes) typically are not standardized, but can still be used to assess children. Criterion-referenced measures identify children's strengths and weaknesses by comparing their performances to a predefined criterion (Shipley \& McAfee, 2015). In other words, children are assessed based on what is expected of them; whether in terms of content area in school, or language.

It refers to how competent the child is to complete a task/domain rather than comparing the child to others in that age group (Fulcher\&Svalberg, 2013).

In addition to standardized and criterion referenced measures, parental report evaluations also provide vital insight into the language strengths and weaknesses of children. Certain parental measures such as rating scales ask caregivers to indicate the severity of certain symptoms and behaviors common amongst children with LI. They can be useful because parents have access to children's behavior across a variety of environments and time periods (Redmond, 2016). A particular parental measure designed to assess social-communicative impairments in daily interactions is the Children's Communication Checklist (CCC-2) (CCC-2, Bishop, 2006).

The CCC-2 was developed to meet three clinical purposes: (1) identify children who may have a language impairment, (2) identify children who may have a pragmatic language impairment, and (3) identify children who may have autism spectrum disorder. This parental measure was designed for children between the ages 4 and 16 whose primary language is English. The CCC-2 contains 70 items grouped in 10 subscales measuring various aspects of communication: language (speech, syntax, semantics, coherence), pragmatics (inappropriate initiation, stereotyped language, the use of context, and non-verbal communication), and two scales assess the behaviors that are affected in children with ASD (social relationships and interests) (Fortea et al., 2018). In a study conducted by Timler (2014), significant correlations were found when the CCC-2 and the CELF-4 scores were compared, suggesting that the CCC-2 can accurately classify students with ADHD and concurrent LI.

Furthermore, studies have shown the CCC-2 to be useful in distinguishing language-impaired children from typically developing children (Vaisanen, Loukusa, Moilanen, \& Yliherva, 2014). Clinical settings may not be the best environment to test children with ADHD because they may be able to concentrate better in a controlled environment, which a clinical setting often maximizes. Therefore, assessments that require information about their behavior in situations other than the testing site can provide vital insight into the everyday language use of children with ADHD (Vaisanen et al., 2014). However, because parents are providing the input, responses are subjective and may be influenced by their particular view or opinion of language impairments. At times, parents may exaggerate or underrate their children's difficulties (Vaisanen et al., 2014).

In a study by Romski et al. (2018), clinician reports of receptive and expressive language capabilities were found to be a reliable assessment in children with developmental delays. They also found that obtaining a holistic description of a child's language abilities is not possible in isolation, but rather must include a combination of measures including standardized tests, parental ratings as well as clinician reports (Romski et al., 2018).

In summary, given the difficulties present in assessing the speech and language skills of children with ADHD, it is important that we investigate the usefulness of supplementing standardized assessment tools with more informal tools. Parental reports may play an important role in obtaining a wider perspective in these children's language abilities not only in diagnostic assessments but also in measuring treatment effectiveness. The current study compared results of a standardized assessment measure (PLS-5) versusa parental report (CCC-2) to determine if both measures would yield comparable results.

\section{Method}

The participants of this study included 12 children ages 4 yrs-10months to 7 yrs-7months $($ Mean age $=5$ yrs-5months) diagnosed with ADHD. Children and their caregivers were recruited from local schools and mental health agencies via brochures, radio and newspaper ads, and open houses/parent workshops. Legal guardians contacted the clinic and were directed to the study staff for screening questions to determine eligibility. If the parent (1) endorsed clinically significant levels of ADHD symptoms (six or more symptoms of either Inattention or Hyperactivity/Impulsivity according to the DSM-5 (APA, 2013) or a previous diagnosis of ADHD), (2) indicated that the child was currently displaying clinically significant academic, behavioral, or social impairments as measured by a score of 3 or higher on a seven-point impairment rating scale (Fabiano et al., 2006), and (3) were not taking any psychotropic medication, the parent and child were invited to participate in an assessment to determine study eligibility. Participants were also required to be enrolled in school during the previous year, have an estimated IQ of 70 or higher, have no confirmed history of an Autism Spectrum Disorder, and be able to attend an 8-week summer treatment program (STPPreK; Graziano et al., 2014) prior to the start of the next school year. 
During intake, ADHD diagnosis (and comorbid disruptive behavior disorders) was assessed through a combination of parent structured interview (Computerized-Diagnostic Interview Schedule for Children; (Shaffer, Fisher, Lucas, Dulcan, \& Schwab-Stone, 2000) and parent and teacher ratings of symptoms and impairment (Disruptive Behavior Disorders Rating Scale, Impairment Rating Scale; Fabiano et al., 2006; Pelham, Gnagy, Greenslade, \& Milich, 1992), as is recommended practice (Pelham, Fabiano, \& Massetti, 2005). Dual Ph.D. level clinician review was used to determine diagnosis and eligibility. Assessment measures of children's intellectual, academic, school readiness and self-regulation skills were also obtained during this initial intake assessment.

As part of the assessments for enrollment in the STP-PreK, children suspected of having a language impairment also received an assessment of speech and language skills that included administration of the Preschool Language Scales $5^{\text {th }}$ Edition (PLS-5; Zimmerman, Steiner, \& Pond, 2013). Those identified with concurrent language impairment received speech-language therapy as part of the STP-PreK. Therapy was administered by speech-language pathology graduate students under the supervision of an experienced, licensed, and certified speech-language pathologist, and goals of intervention were closely tied to performance on PLS-5 items that were missed by each participant. A subset of children receiving speech-language therapy participated in this study.

During the initial evaluation or within the first week of the STP-PreK, parents were asked to complete the CCC-2 (Bishop, 2006). The STP-PreK, which is an 8-week multimodal interventionto improve behavioral, socio-emotional, and academic readiness for children prior the kindergarten transition (Graziano et al., 2014). The behavior modification program included the use of a point system, daily report card,time-out system, social reinforcement, and daily and weekly rewards. The socioemotional curriculum consisted of social skills, emotional awareness, and self-regulation training via in-vivo training, the use of puppets, and reinforcement of the skills throughout the day. Parents also attended eight 2-hour weekly group parenting sessions based on the School Readiness Parenting Program (SRPP) (Graziano, Ros, Hart, \& Slavec, 2018). The children included in this study received two 30-minute individual speechlanguage therapy sessions and 60 minutes of group language therapy within the classroom per week for the 8-week duration of the program. At the final week of the program, the PLS-5 was re-administered and parents were asked to complete the CCC-2 a second time. Parents did not have access to their previous responses to the CCC-2. All responses on the CCC-2 were entered into the computerized scoring program included with the inventory for further analysis. Results of pre- and post-test scores on the PLS-5 and CCC-2 were compared using paired t-tests.

\section{Results}

Paired t-tests showed that PLS-5 scorespost-interventionwere significantly improved as compared to pre-intervention with a mean of $101.5(\mathrm{SD}=8.4)$ post-intervention and $86.16(\mathrm{SD}=6.1)$ pre-intervention $(\mathrm{t}=9.27, \mathrm{p}=.001)$. The same was true for CCC-2 composite scores with a mean of $93.4(\mathrm{SD}=12.3)$ post-intervention and $86.75(\mathrm{SD}=13.1)$ preintervention ( $\mathrm{t}=3.337, \mathrm{p}=0.003)$. Conversely, paired $\mathrm{t}$-tests showed no significant difference when comparing PLS-5 and CCC-2 scores pre-intervention with means of $86.16(\mathrm{SD}=6.1)$ and $86.75(\mathrm{SD}=13.1)$ respectively $(\mathrm{t}=0.139$, $\mathrm{p}=0.445)$, but a significant difference post-intervention with a mean of $101.5(\mathrm{SD}=8.4)$ for the PLS-5 and $93.4(\mathrm{SD}=$ 12.4) for the CCC $-2(t=-1.86, \mathrm{p}=0.037)$.

As explained previously, the CCC-2 contains 70 items grouped in 10 subscales measuring different areas of communication, which are further grouped into language (speech, syntax, semantics, coherence), pragmatics (initiation, stereotyped language, the use of context, and non-verbal communication), and behaviors (social relationships and interests). These subscales produce standardized scaled scores with a mean of 10 and standard deviation of 3. Paired ttests performed for each subscales comparing pre- and post-intervention scaled scores showed significant improvements in the areas of speech $(\mathrm{t}=1.77, \mathrm{p}=.05)$, syntax $(\mathrm{t}=2.03, \mathrm{p}=.03)$, semantics $(\mathrm{t}=2.15, \mathrm{p}=.02)$, and social interaction ( $\mathrm{t}=2.01, \mathrm{p}=.03)$. The following areas yielded non-significant results: cohesion, initiation, script, context, interest and nonverbal communication. Table 1 shows Mean scores for each area pre- and post-intervention. 


\begin{tabular}{|l|l|l|l|l|}
\hline AREA & PRE & POST & $\begin{array}{l}\text { T- } \\
\text { VALUE }\end{array}$ & P-VALUE \\
\hline Speech* & 6.92 & 8.17 & 1.77 & 0.05 \\
\hline Syntax* & 7.58 & 8.75 & 2.03 & 0.03 \\
\hline Semantics* & 6.83 & 8.67 & 2.15 & 0.02 \\
\hline Cohesion & 7.75 & 8.66 & 1.27 & 0.11 \\
\hline Initiation & 8.42 & 9.33 & 1.05 & 0.15 \\
\hline $\begin{array}{l}\text { Scripted } \\
\text { Language }\end{array}$ & 10.25 & 9.33 & 1.16 & 0.13 \\
\hline Context & 8.66 & 8.25 & 0.57 & 0.28 \\
\hline Nonverbal & 7.92 & 9.16 & 1.48 & 0.08 \\
\hline Interaction* & 9.08 & 9.75 & 2.01 & 0.03 \\
\hline Interests & 9.08 & 9.75 & 0.95 & 0.17 \\
\hline
\end{tabular}

Table 1. Mean subarea scores pre- and post-intervention with associated t-values and probabilities

\section{Discussion}

This study attempted to determine whether a parental report such as the CCC-2 and a standardized test, such as the PLS-5, provide equivalent measures of language improvement after intervention in preschoolers with concurrent language impairment and ADHD. Results indicated that both measures showed significant improvements in the children's language skills. However, the PLS-5 showed more pronounced improvement than the CCC-2. This difference is probably a result of the goals addressed in therapy being directly related to deficits shown on the PLS-5. For example, if a child was not able to identify and produce pronouns appropriately for his/her age, the clinician targeted this goal in treatment. Upon re-administration of this same test item, children were more likely to respond correctly. A limited set of the goals were obtained for each child based on items missed on the PLS-5, which in turn assesses a limited number of specific skills expected to be acquired by preschoolers. In contrast, the CCC-2 is a noncriterion-referenced questionnaire that categorizes the child's language abilities based on broader skills observed by parents in diverse environments. Not only did the CCC-2 not influence the goals set for treatment, but it arguably assesses more generalized communication skills necessary for daily living. Such generalized improvement would be expected to take longer than a limited set of specific skills.

It is remarkable to note how closely the results of the CCC-2 compared to the PLS-5 results pre-intervention, with means of 86.75 and 86.16 respectively. Assuming that the PLS-5 is a valid and reliable measure of language abilities in preschoolers as suggested by Denman et al. (2017), parents were very accurate in estimating their children's language abilities. By the same token, parents noticed significant improvement in their children's abilities post-intervention, but did not seem to overestimate their improvement as their ratings were significantly below improvement in PLS-5 scores (Mean score for CCC-2 was 93.4 versus 101.5 for the PLS-5). It is safe to assume that the PLS-5 scores postintervention were slightly inflated, although similarly falling in the average range of functioning, given that goals addressed in therapy were directly related to specific test items missed on the PLS-5.

A closer inspection of the subareas assessed by the CCC-2 revealed that not all areas improved equally. Significant improvement was reported for the areas of Speech, Syntax, and Semantics, all areas that are traditionally targeted in speech and language therapy. Each child received 2 hours of therapy a week, including 1 hour of traditional individual "pull-out" therapy and 1 hour of "push-in" intervention, where the clinician worked with a group of children within their classroom. Goals of speech, syntax, and semantics were addressed in individual therapy and reinforced within the broader classroom environment. The other subarea that showed significant improvement was Social Interaction, which was also addressed in pragmatic goals within individual therapy as well as in group "push-in" sessions and throughout the day as part of the regular curriculum of the summer program. Again, it is important to note that parents did not indiscriminately give their children higher scores post-intervention. There were 6 subareas that did not show statistically significant improvement according to parents' reports (cohesion, initiation, script, context, interest and nonverbal). It is clear that parents were able to perceive significant communication improvement particularly in areas that were addressed in speech-language therapy. 
Other research studies have indicated parental reports are useful to test children with ADHD who may perform better in a controlled clinical setting as opposed to their everyday language use (e.g., Vaisanen et al., 2014). However, studies have also indicated that parental report measures may be influenced by subjective bias of the respondent (Bishop, 2006). It is possible that parents exaggerated or underestimated their children's difficulties perhaps influencing the results obtained via the CCC-2 both pre- and post-intervention. Nonetheless, our results suggest that parents can be reliable informants on their children's communication abilities. Parental reports can be particularly useful when combined with other data obtained, such as in standardized assessments, teacher reports, and clinical observations, and they can be used not only in diagnostic assessments but also for measuring progress.

There are obvious limitations to this study. Using the same standardized assessment to test language skills before and after intervention likely caused improved performance from retaking the same test in such a short amount of time between administrations. Roediger and Karpicke (2006) described the direct effects of repeated testing on retention across various experiments in their review of past research findings. They saw improved performance of the participants as the post-test assessed the same test items, thus weakening external validity. Varying the test materials or using an alternate test form to assess language skills in the pre- and post-test design would have increased validity of our study. In addition, the therapy provided essentially targeted the areas in the PLS-5 where the participant displayed the most deficits. Therefore, the goals of therapy provided an intensive preparation for the re-administration of the PLS-5. In the future, a different standardized test could be administered post treatment and compared to both the results of the PLS-5 and CCC-2 to provide stronger evidence of improved performance and of the accuracy of parental reports.

Another obvious limitation of this study is the small number of participants. Future research of this topic should include a larger sample size as well as other clinical populations (e.g., children with Autism Spectrum Disorder) who present with language impairments. Our participants were chosen via convenience sampling, reducing generalization of results as randomization was not possible. Further studies should include random sampling, which would improve validity, minimize confounding variables, and reduce biases.

\section{Acknowledgements}

The research reported here was supported by grants from the National Institute of Mental Health (R01MH112588) and the National Institute of Diabetes and Digestive and Kidney Diseases (R01DK119814), to the last author as well as a local grant from The Children's Trust (1329-7290) to the third and last authors. The opinions expressed are those of the authors and do not represent views of NIH or The Children's Trust. We would also like to acknowledge the support of Miami-Dade County Public Schools and thank the families and dedicated staff who participated in the study.

\section{References}

American Psychiatric Association. (2013). Diagnostic and statistical manual of mental disorders(5th ed.). Washington, DC: American Psychiatric Publishing.

American Speech-Language Hearing Association. (2008). Roles and Responsibilities of Speech-Language Pathologists in Early Intervention: Guidelines. Retrieved from http://www.asha.org/policy/GL2008-00293.htm

Armstrong, M.B \& Nettleton, S.K. (2004). Attention deficit hyperactivity disorder and preschool children. Seminars in Speech and Language. 25(3):225-32.

Barkley, R. A. (Ed.). (2014). Attention-deficit hyperactivity disorder: A handbook for diagnosisand treatment. Guilford Publications.

Bickford-Smith, A., Wijayatilake, L., \& Woods, G. (2005). Evaluating the effectiveness of anearly years language intervention. Educational Psychology in Practice, 21(3), 161-173.doi: 10.1080/02667360500205859.

Bishop, E. (2006). Children's Communication Checklist-2 (United States Edition). SanAntonio, TX: Harcourt Assessment, Inc.

Broomfield, J. \& Dodd, B. (2011). Is Speech and Language Therapy Effective for Children with $\quad$ Primary Speech and Language Impairment? Report of randomized control trial. International Journal of Language and Communication Disorders, 46(6) 628-640.

Crais, E. R. (2011). Testing and beyond: Strategies and tools for evaluating and assessing infantsand toddlers. Language, Speech, and Hearing Services in Schools, 42(3), 341-364.

Denman, D., Speyer, R., Munro, N., Pearce, W. M., Chen, Y. W., \& Cordier, R. (2017). Psychometric properties of language assessments for children aged 4-12 years: A systematic review. Frontiers in Psychology, 8, 1515.

Docking, K., Munro, N., Cordier, R., \& Ellis, P. (2013). Examining the language skills ofchildren with ADHD following a play-based intervention. Child Language Teaching and Therapy, 29(3), 291-304.

DuPaul, G.J., Weyandt, L.L., \& Janusis, G.M. (2011). ADHD in the classroom: Effectiveintervention strategies. Theory Into Practice, 50, 35-42. doi:10.1080/00405841.2011.534935. 
Fortea, I. B., Forner, C. B., Colomer, C., Casas, A. M., \& Miranda, B. R. (2018). Communicative skills in Spanish children with Autism Spectrum Disorder and children with Attention Deficit Hyperactivity Disorder. Analysis through parents' perceptions and narrative production. Research in Autism Spectrum Disorders, 50, 22-31. doi: 10.1016/j.rasd.2018.02.006

Fulcher, Glenn \& Svalberg, Agneta. (2013). Limited aspects of reality: Frames of reference in language assessment. International Journal of English Studies. 13. 10.6018/ijes.13.2.184061

Graziano, P., Slavec, J., Hart, K., Garcia, A., \& Pelham, W. (2014). Improving school readiness in preschoolers with behavior problems: Results from a summer treatment program. Journal of Psychopathology and Behavioral Assessment, 36(4), 555-569.

Kamphaus, R.W. (2015). Behavior Assessment System for Children, Second Edition (BASC- 2). In The Encyclopedia of Clinical Psychology (eds R.L. Cautin and S.O. Lilienfeld). doi:10.1002/9781118625392.wbecp447

Law, J., Garrett, Z., \& Nye, C. (2004). The efficacy of treatment for children with developmentalspeech and language delay/disorder: A meta-analysis. Journal of Speech, Language, andHearing Research, 47, 924-943. doi: 10924388/04/4704-0924.

Oram, J., Fine, J., Okamoto, C., \& Tannock, R. (1999). Assessing the Language of Children withAttention Deficit Hyperactivity Disorder. American Journal of Speech-LanguagePathology, 8, 72-80.

Redmond, S. M. (2016). Language impairment in the attention-deficit/hyperactivity disorder context. Journal of Speech, Language, and Hearing Research, 59(1), 133-142. doi:10.1044/2015_JSLHR-L-15-0038

Redmond, S. M., Ash, A. C., \& Hogan, T. P. (2015). Consequences of Co-OccurringAttention-Deficit/Hyperactivity Disorder on Children's Language Impairments. Language, speech, and hearing services in schools, 46(2), 6880.

Roediger, H. L., \& Karpicke, J. D. (2006). The power of testing memory: Basic research and implications for educational practice. Perspectives on Psychological Science, 1(3), 181-210.

Romski, M. et al. (2018). Language Assessment for Children With a Range of Neurodevelopmental Disorders Across Four Languages in South Africa. American Journal of Speech-Language Pathology, 27, p. 602-615.

Roulstone, S., Wren, Y., Bakopoulou, I., \& Lindsay, G. (2012). Interventions for children withspeech, language and communication needs: An exploration of current practice. ChildLanguage Teaching and Therapy, 28(3), 325341. doi: 10.1177/0265659012456385.

Sciberras, E., Mueller, K.L., Efron, D., Bisset, M., Anderson, V. Schilpzand, E.J., Jongeling, B., \& Nicholson, J.M. (2014). Language problems in children with ADHD: A community-based study. Pediatrics. 133 (5), 793-800.

Semel, E., Wiig, E. H., \& Secord, W. A. (2004). Clinical Evaluation of Language Fundamentals—Fourth Edition Screening Test: Examiner's manual. San Antonio, TX: Pearson Assessment.

Shipley, K., \& McAfee, J. (2015). Assessment in speech-language pathology: A resourcemanual. Cengage learning.

Timler, G. R. (2014). Use of the Children's Communication Checklist - 2 for Classification of Language Impairment Risk in Young School-Age Children With Attention-Deficit/Hyperactivity Disorder. American Journal of Speech-Language Pathology, 23(1), 73-83. https://doi-org.ezproxy.fiu.edu

Väisänen, R., Loukusa, S., Moilanen, I., \& Yliherva, A. (2014). Language and pragmatic profile in children with ADHD measured by Children's Communication Checklist 2nd edition. Logopedics Phoniatrics Vocology, 39(4), 179-187. doi: 10.3109/14015439.2013.784802

Wake, M., Tobin. S., Levickis P., Gold L., Ukoumunne O.C., Zens, N., Goldfeld, S., Le, H.,

Law, J., \& Reilly S. (2013). Randomized trial of a population-based, home-deliveredintervention for preschool language delay. Pediatrics. 132(4), 895- 902.

Wiig, E. H., Semel, E., \& Secord, W. A. (2013). Clinical Evaluation of LanguageFundamentals-Fifth Edition (CELF5). Bloomington, MN: NCS Pearson.

Zimmerman, I.E., Steiner, V.G., \& Pond, R.E. (2013). Preschool Language Scales, Fifth Edition (PLS-5). San Antonio, TX: The Psychological Corporation. 